\title{
NEW ECTOPARASITIC ABROLOPHINE MITES (ACARI, ERYTHRAEIDAE, MARANTELOPHUS) ON THRIPS AND APHIDS (INSECTA) FROM IRAN
}

\author{
Masoud Hakimitabar ${ }^{1}$, Hamed Ghobari ${ }^{2}$ and Alireza Saboori ${ }^{3}$ \\ ${ }^{1}$ Department of Plant Protection, College of Agriculture \\ University of Shahrood, Shahrood, Iran; E-mail: hakimitabar@yahoo.com \\ ${ }^{2}$ Department of Plant Protection, College of Agriculture \\ University of Kurdistan, Sanandaj, Iran; E-mail: ghobari@gmail.com \\ ${ }^{3}$ Department of Plant Protection, Faculty of Agriculture \\ University of Tehran, Karaj, Iran; E-mail: saboori@ut.ac.ir
}

Larvae of Marantelophus sanandajensis Hakimitabar et Saboori sp. n. collected from Kurdistan and Alborz provinces are described and illustrated as ectoparasite of aphids and thrips. A key to the species of Marantelophus of the world is presented.

Key words: Trombidiformes, larva, Thysanoptera, Aphididae, Iran.

\section{INTRODUCTION}

There are four genera (Abrolophus Berlese, 1981, Grandjeanella Southcott, 1961, Nagoricanella Haitlinger, 2009 and Marantelophus Haitlinger, 2011) in the subfamily Abrolophinae (Trombidiformes: Prostigmata: Erythraeidae). Nagoricanella have characters that comply with definition of subfamily Abrolophinae so MąKol and WohLtmann (2012) have cited it mistakenly in Phanolophinae.

Larvae of most species in this subfamily are ectoparasites on Homoptera and Thysanoptera (Insecta) or display a predatory life-style (WoHLtmanN 2000, 2001).

The genus Marantelophus is known by the larval stage only and presently includes eight species: M. alaperti Haitlinger, 2011 from Indonesia; M. emanueli (Haitlinger, 2010) from Turkey; M. ainae (Haitlinger, 2002) from Spain; M. kamalii (Saboori et Atamehr, 2000) from Iran; M. haitlingeri (Goldarazena et Zhang, 1997) from Spain; M. multisetosa (Zhang et Goldarazena, 1996) from Croatia, Greece, Hungary, Romania, San Marino, Spain, Turkey and Ukraine; M. bella (Zhang, 1996) from Iran and Turkey; M. ostovani (Haitlinger et Saboori, 1996) from Iran. Among them, M. kamalii and M. ostovani have been described from Iran (Zhang \& Goldarazena 1996, Haitlinger \& Saboori 1996, Goldarazena \& Zhang 1997, Saboori \& Atamehr 2000, Haitlinger 2002, 2010, 2011, MąкоL \& Wohltмann 2012). Parasitic associations of larvae are listed in Table 4. 
In this paper, we describe the larva of Marantelophus sanandajensis Hakimitabar et Saboori sp. n. ectoparasitic on an unidentified Thysanoptera (Insecta) and Aphididae (Insecta) from Karaj and Sanandaj, Iran.

\section{MATERIAL AND METHODS}

Thysanoptera and Aphididae were collected by an insect net or by shaking the foliage on white tray and subsequently preserved in $70 \%$ ethanol. Mite specimens were detached by an insect pin cleared in Nessbitt's fluid and mounted in Faure medium (Walter \& Krantz 2009). Figures were drawn and measurements were calculated using a BX51 Olympus microscope equipped with a drawing tube and magnification changer. Terminology and abbreviations follow SABOORI et al. (2009). Measurements are given in micrometers $(\mu \mathrm{m})$.

\section{Marantelophus sanandajensis Hakimitabar et Saboori sp. n.} (Figs 1-9)

Diagnosis - Ti III < 80; fn TFe 8-6-6; fn Ti I-III 15-13-13; with 2 fine setulose setae on palp-tarsus.

Description (holotype) - Idiosoma oval, dorsal surface with 60 (for paratypes see Table 2) barbed and pointed setae (Fig. 1), 2 pairs of setae anterior to eyes. Scutum pentagonal, punctate and wider than long (except in paratypes $\mathrm{d} \& \mathrm{e}$ ), lateral parts of anterior border rounded and its median part concave, anterolateral and posterolateral borders straight, posterior line convex in median, scutum with 2 pairs of scutalae; PL longer than AL; both with barbs and pointed. Posterior pair of sensilla (S) approximately twice as long as anterior pair (AM), both barbed at posterior half and pointed (Fig. 1). Each side of scutum with one eye, circular, not on platelets; 16 in diameter.

Ventral surface of idiosoma with sternalae I, II and III (1a, 2a \& 3a), 4 barbed setae between coxae I \& II, 16 barbed setae between coxae II and III (for paratypes see Table 2) and 22 setae behind coxae III (for paratypes see Table 2), all barbed and pointed. Sternalae $1 a$ as long as $2 a$ (for paratypes see Table 2 ), both longer than sternalae $3 a$. Sternalae I- III barbed and pointed. Coxalae $1 b$ longer than other coxalae; coxalae $2 b$ longer than coxalae $3 b$; all coxalae barbed and pointed (Fig. 2). NDV $=60+38=98$ (not clear, 91, $113 \& 104$ in paratypes $1 \mathrm{~b}-1 \mathrm{e})$.

Gnathosoma with nude galealae $(\mathrm{Ga})$ and two pairs of nude hypostomalae, posterior hypostomalae (pHy) (23) longer than anterior hypostomalae (aHy) (9). Palpal femur and genu each with 2 barbed and pointed setae (Fig. 3). Tibia with 2 barbed and one claw-like setae. Tarsus with 8 setae including 2 barbed and 4 nude normal setae, a solenidion and an eupathidium (Fig. 3). $\mathrm{fPp}=0-\mathrm{BB}-\mathrm{BB}-\mathrm{BBN}_{2}-\mathrm{NNNNBB} \omega \zeta$. Palps, ventral gnathosoma and cheliceral bases punctate. Supracoxal seta of palp $(e P)$ peg like, 4 long.

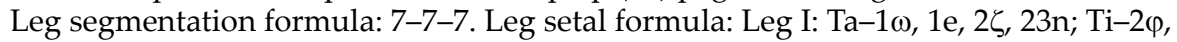

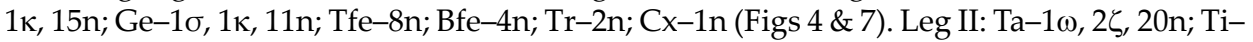

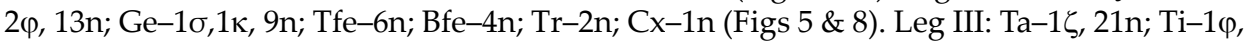
13n; Ge-16, 9n; Tfe-6n; Bfe-4n; Tr-2n; Cx-1n (Figs 6 \& 9). Tarsi with two falciform claws and a thin claw-like empodium. Measurements are given in Table 1.

Type material. The holotype (ARS-20140620-1a) and paratypes (ARS-20140620-1b \& 1c) were collected by M. Hakimitabar, ectoparasitic on unidentified Thysanoptera and 

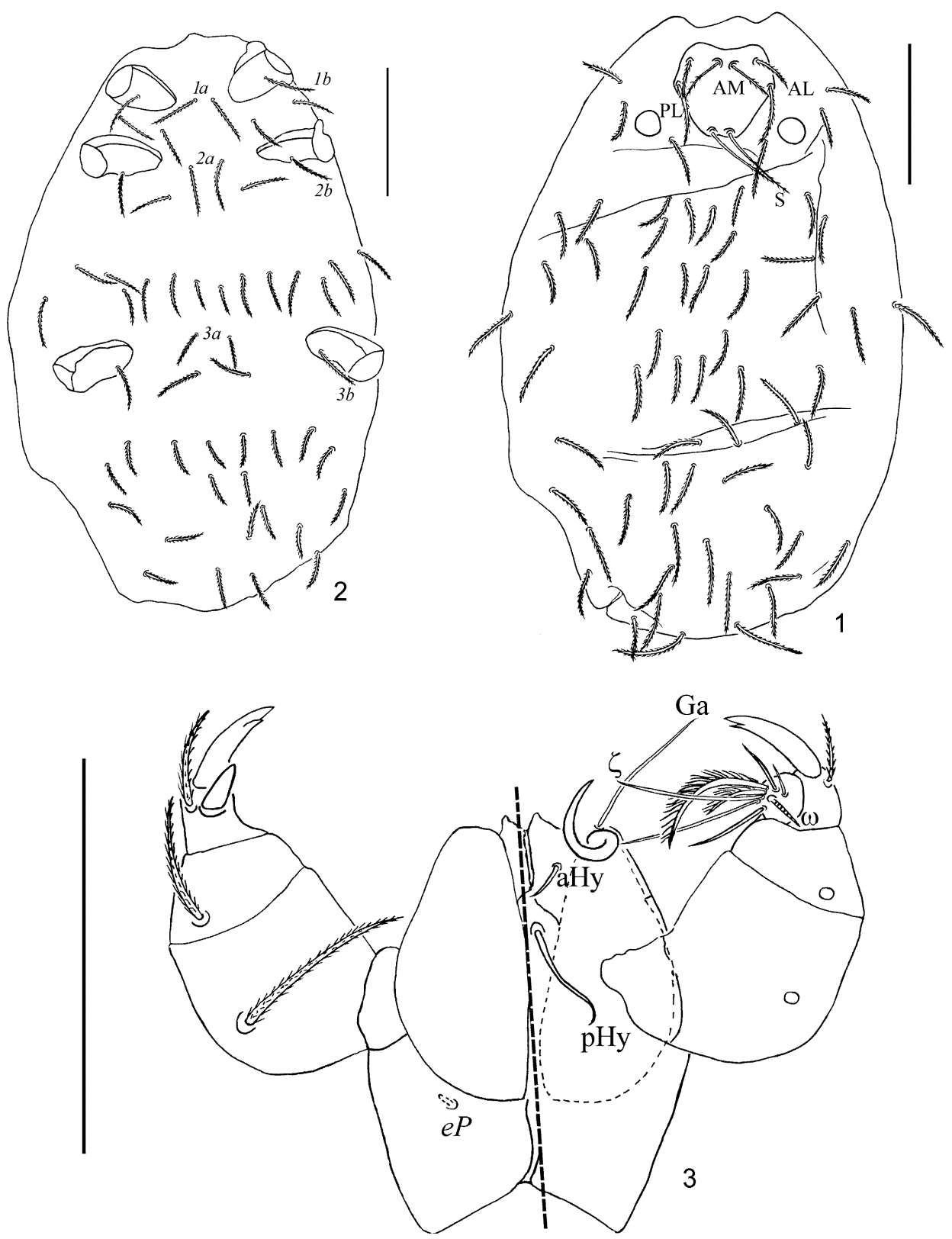

Figs 1-3. Marantelophus sanandajensis Hakimitabar et Saboori sp. n. (larva): 1 = dorsal view of idiosoma, 2 = ventral view of idiosoma, 3 = ventral view (right) and dorsal view of gnathosoma (left). Scale bars: $100 \mu \mathrm{m}$. 


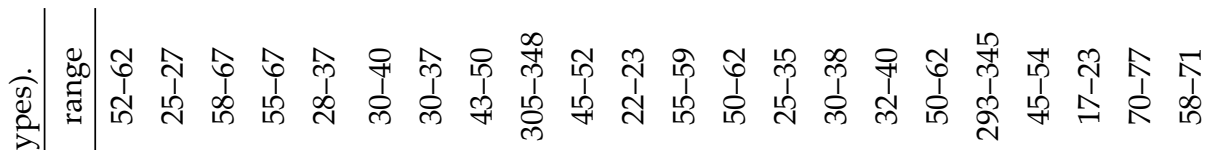

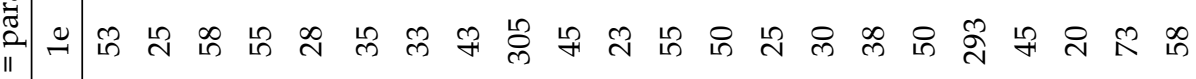

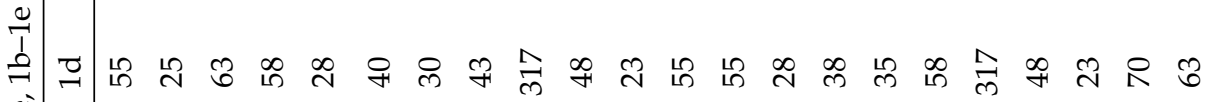
帘 跑 규 त्र 㓂

:

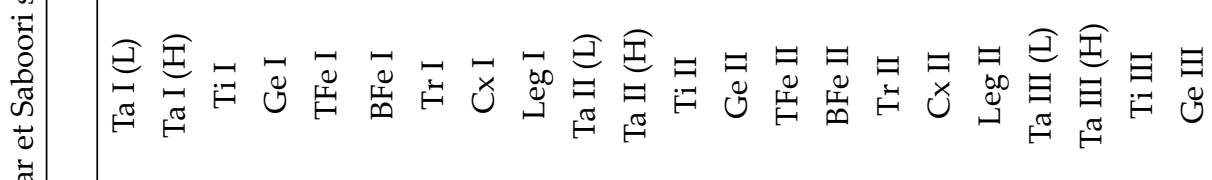

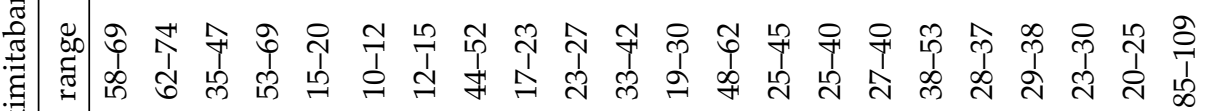
离 焉 豙

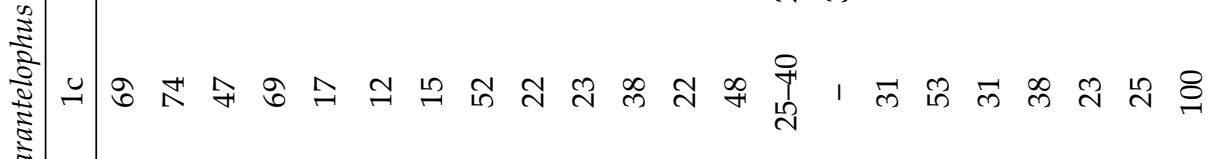

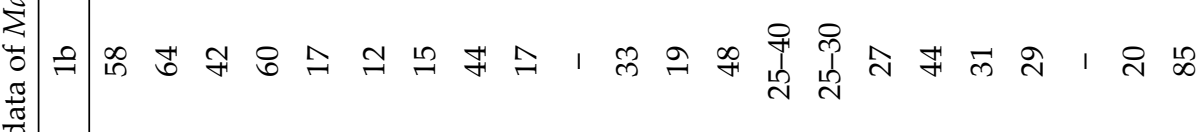

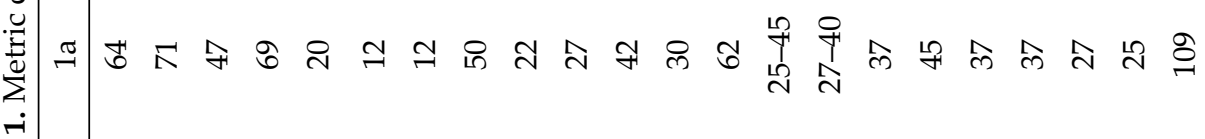
音

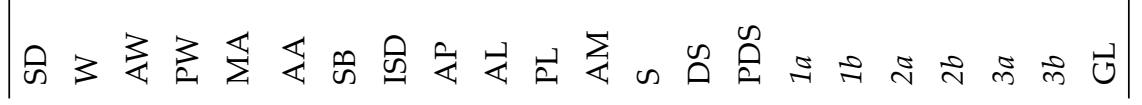




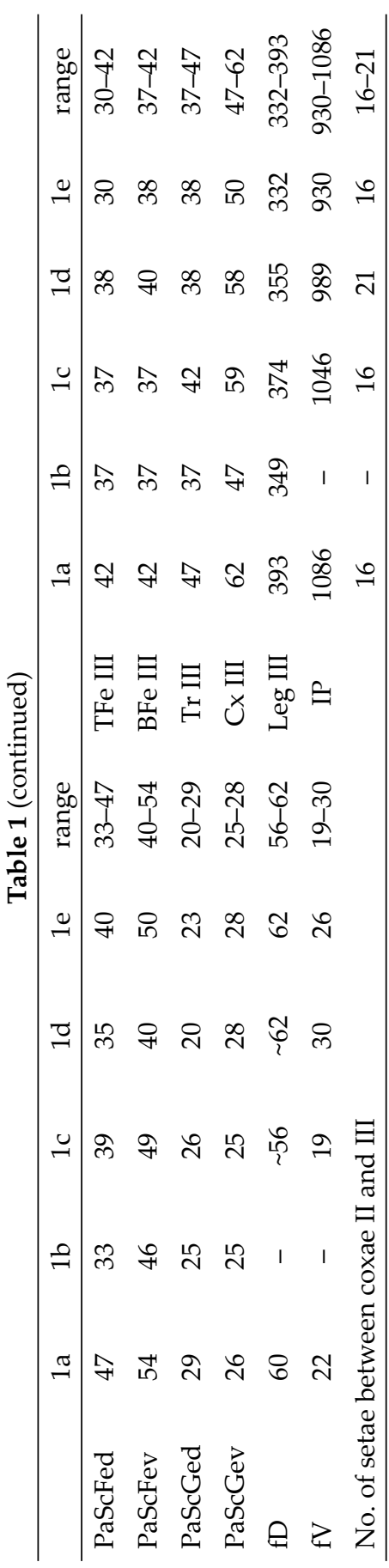

Aphididae, 22nd June 2009 in Koushk Bala village, Chalous road, Karaj, Iran (35 $59.057^{\prime} \mathrm{N} 51^{\circ} 5.296^{\prime} \mathrm{E}$, $1824 \mathrm{~m}$ a.s.l.). Other paratypes (ARS-014620-1d \&1e) were collected by F. Afrasiabi and H. Ghobari as ectoparasite of unknown Aphididae on alfalfa (Medicago sativa), 30 May 2007, in Kouleh Sareh village, Sanandaj, Iran $\left(46^{\circ} 53^{\prime} 32^{\prime \prime} \mathrm{N}, 5^{\circ} 0^{\prime} 32^{\prime \prime} \mathrm{E}, 1788\right.$ $\mathrm{m}$ a.s.l.). The holotype and two paratypes (ARS2014620-1b \& 1d) are deposited in the Acarological Collection, Jalal Afshar Zoological Museum, Faculty of Agriculture, University of Tehran, Karaj, Iran. Two paratypes (ARS-2014620-1c \& 1e) are deposited in the Acarological Collection, Acarological Society of Iran, Karaj, Iran.

Etymology. Name of this species refers to the locality of some paratypes, Sanandaj city, Iran.

Remarks. There are eight species in this genus. Marantelophus sanandajensis Hakimitabar et Saboori sp. n. differs from $M$. alaperti in the longer ISD (44-52 vs. 38), shorter AL (23-27 vs. 58), PL (33-42 vs. 82), GL (85-109 vs. 140$)$, Ta I (52-62 vs. 80$)$, Ta III (45-54 vs. 84), Ti III (70-77 vs. 112), fn TFe I-III (8-6-6 vs. $7-5-5)$, fn Ti I-III (15-13-13 vs. 12-12-12), fn Ta I-III (23-20-21 vs. 17-15-18), number of setae on Ge I (11 vs. 9), fD (60 vs. 96); from M. ostovani in the shorter AL (23-27 vs. 56), Ti (58-67 vs. 82), Ti II (55-59 vs. 84), Ti III (70-77 vs. 102), Ge III (58-71 vs. 90), number of setae on BFe I (4 vs. 3), fn TFe I-III (8-6-6 vs. 7-5-5), number of setae on Ge II (9 vs. 8), ), fn Ti I-III (15-13-13 vs. 14-15-10), fn Ta I-III (23-20-21 vs. 26-19-18); from $M$. haitlingeri in the shorter AW (35-47 vs. 70), PW (53-69 vs. 82$)$, AL (23-27 vs. 54), PL (33-42 vs. 67), AM (17-30 vs. 46), L (58-69 vs. 83), W (60-74 vs. 86$)$, Ta I (52-62 vs. 100), Ti I (58-67 vs. 106), Ge I (55-67 vs. 90), Ta II (45-52 vs. 84$)$, Ti II (55-59 vs. 100), Ge II (50-62 vs. 78), Ta III (45-54 vs. 90), Ti III (70-77 vs. 140), Ge III (58-71 vs. 96), fn BFe I-III (4-4-4 vs. 5-5-5), number of setae on TFe II \& III (6 vs. 5), num- 
ber of setae on Ti I ( 15 vs. 14), fD (60 vs. 119); from M. multisetosa in the shorter Ta II (45-52 vs. 63-65), Ta III (45-54 vs. 66-72), fn BFe I-III (4-4-4 vs. 5-5-5), number of setae on TFe II \& III (6 vs. 5), fn Ti I-III (15-13-13 vs. 13-13-11), fn Ta I-III (23-20-21 vs. 25-20-22), fD (60 vs. $>140)$; from M. bella in the shorter SB (12-15 vs. 36$)$, AM (17-30 vs. 52), Ta I (52-62 vs. 71$)$, Ti I (58-67 vs. 89), Ta II (45-52 vs. 66), Ti II (55-59 vs. 79), Ta III (45-54 vs. 73), Ti III (70-77 vs. 112), fn TFe I-III (8-6-6 vs. 9-9-8), fn Ge I-III (13-10-10 vs. 11-9-9), fn Ti I-III (15-1313 vs. 19-17-16); from M. kamalii in the shorter AL (23-27 vs. 36), GL (85-109 vs. 124-192), Ta I (52-62 vs. 74-77), Ta II (45-52 vs. 66-74), Ta III (45-54 vs. 7477), Ti III (70-77 vs. 96-102), fn BFe I-III (4-4-4 vs. 5-5-5), number of setae on
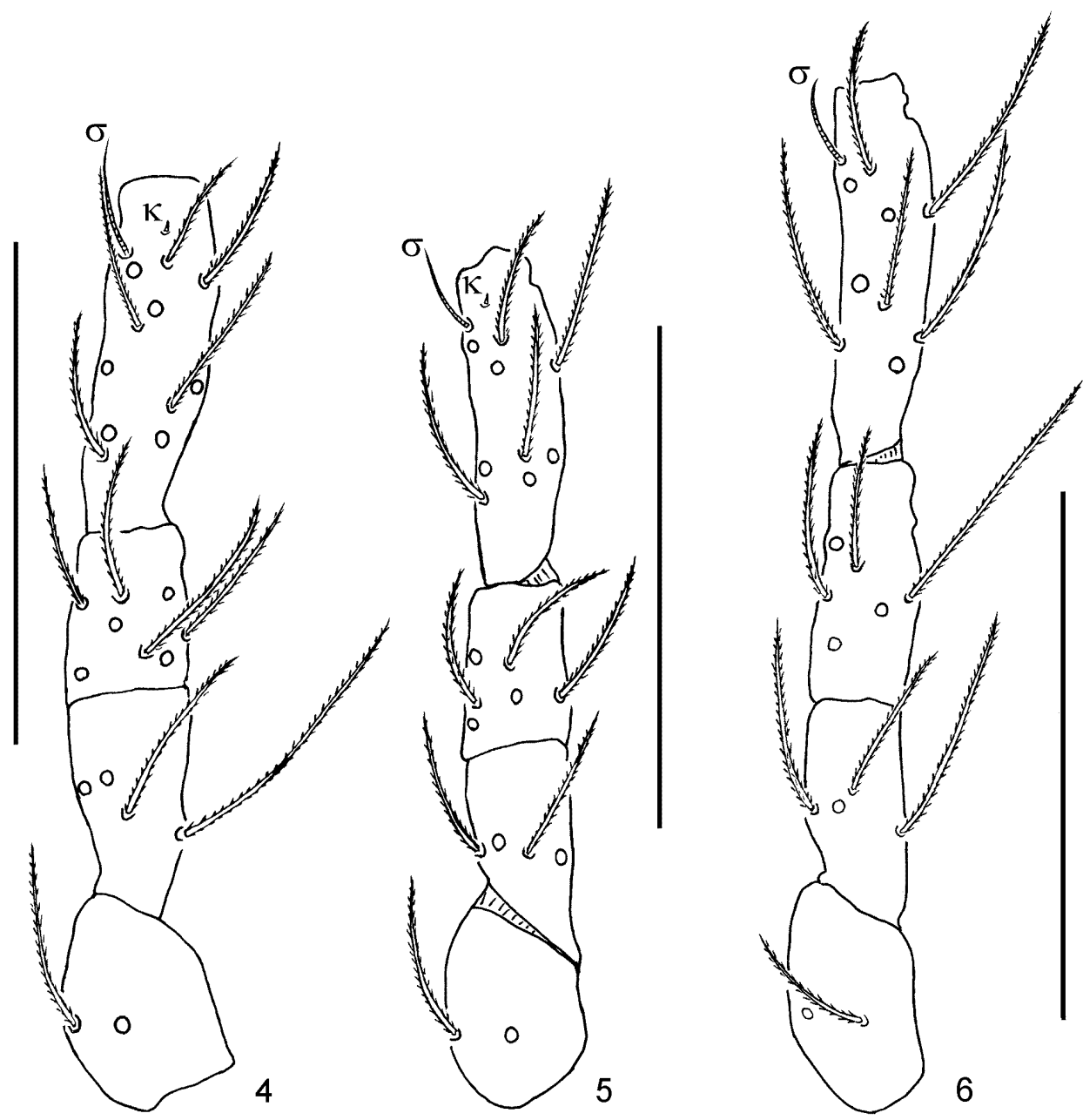

Figs 4-6. Marantelophus sanandajensis Hakimitabar et Saboori sp. n. (larva): $4=\mathrm{Tr}-\mathrm{Ge} \mathrm{I}, 5=$ Tr-Ge II, 6 = Tr-Ge III. Scale bars: $100 \mu \mathrm{m}$. 
TFe II \& III (6 vs. 5), number of setae on Ti I (15 vs. 13), fn Ta I-III (23-20-21 vs. 21-22-22), fD (60 vs. 120 ); from $M$. ainae by SD/W (0.9-1.04 vs. 1.12-1.13), fn TFe I-III (8-6-6 vs. 7-5-5), fn Ge I-III (11-9-9 vs. 10-9-8), fn Ti I-III (15-13-13 vs. 12-12-12), fn Ta I-III (23-20-21 vs. 18-12-14) and from M. emanueli by fn TFe I-III (8-6-6 vs. 5-5-5), fn Ge I-III (11-9-9 vs. 8-8-8), fnTi I-III (15-13-13 vs. 10-10-10), fn Ta I-III (23-20-21 vs. 15-13-17) (see Tables 2 \& 3).

\section{KEY TO LARVAL SPECIES OF MARANTELOPHUS OF THE WORLD}

1 fn Ge 11-9-9

- $\quad$ fn Ge otherwise

2 fn BFe 4-4-4, fnTFe 8-6-6

M. sanandajensis sp. n.

- $\quad$ fn BFe 5-5-5, fnTFe 8-5-5

3 Ti III $>135$, Ti I with 14 normal setae M. haitlingeri

- $\quad$ Ti III < 110, Ti I with 13 normal setae
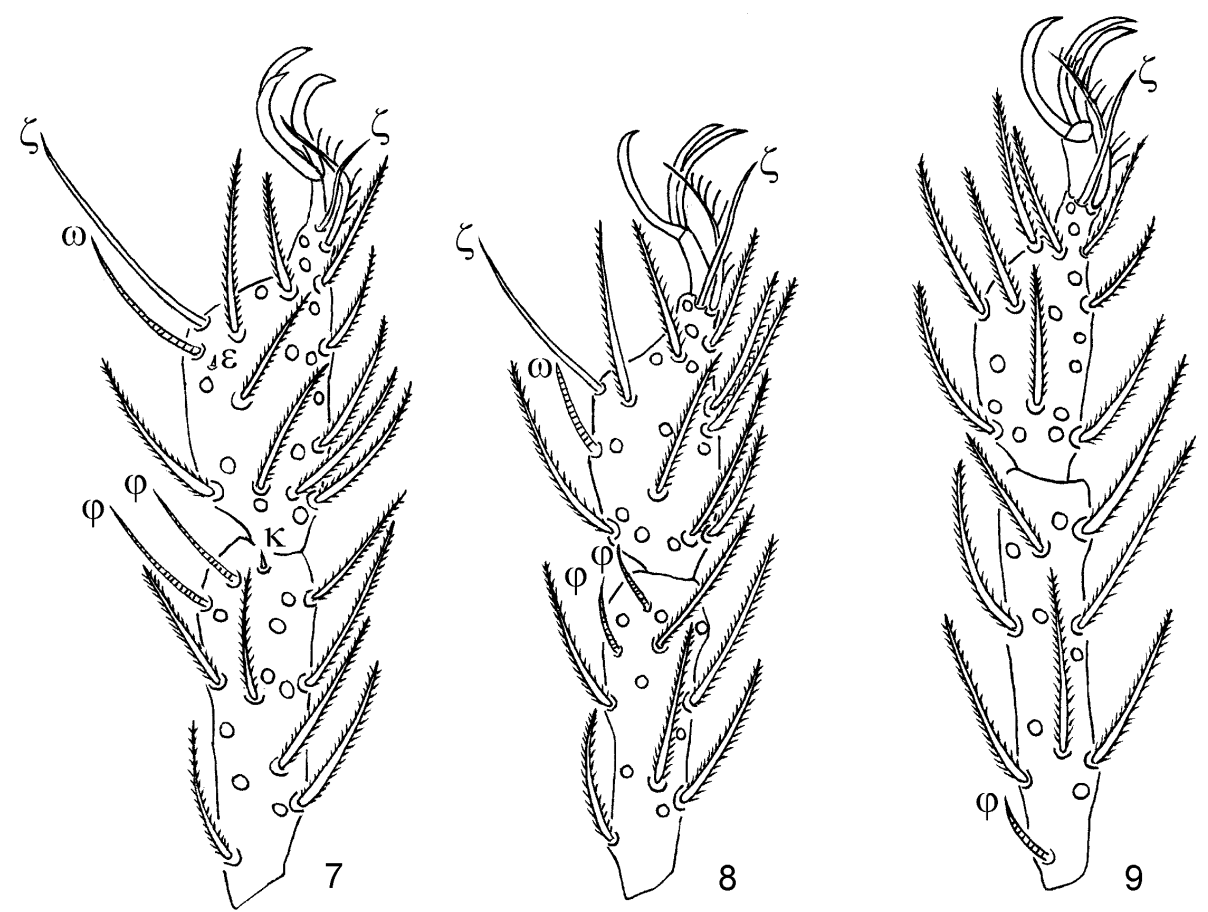

Figs 7-9. Marantelophus sanandajensis Hakimitabar et Saboori sp. n. (larva): $7=\mathrm{Ti}$ and Ta I, $8=\mathrm{Ti}$ and Ta II, $9=\mathrm{Ti}$ and Ta III. Scale bar: $100 \mu \mathrm{m}$. 
4 Ti III with 13 normal setae

M. kamalii

- $\quad$ Ti III with 11 normal setae

M. multisetosa

5 fn Ge 8-8-8

M. emanueli

- $\quad$ fn Ge otherwise

\section{6}

6 fn TFe 9-9-8

M. bella

- $\quad$ fn TFe 7-5-5

7 fn Ti 14-15-10

M. ostovani

- $\quad$ fn Ti 12-12-12

8 Ge I \& III with 9 normal setae, Ti III $>110$

M. alaperti

- $\quad$ Ge I \& III with 10 \& 8 normal setae, respectively, Ti III $<90$

M. ainae

(see Tables 2 \& 3) (Zhang \& Goldarazena 1996, Haitlinger \& Saboori 1996, Goldarazena \& Zhang 1997, Saboori \& Atamehr 2000, Haitlinger 2002, 2010, 2011).

Table 2. Number of setae on leg segments of nine species of the genus Marantelophus: $1=$ M. alaperti, $2=$ M. ostovani, $3=$ M. haitlingeri, $4=$ M. multisetosa, $5=$ M. bella, $6=$ M. kamalii,

\begin{tabular}{llllllllll}
\multicolumn{7}{c}{7} & M. ainae, $8=$ M. sanandajensis & sp. . 9. M. emanueli. \\
\hline Character & 1 & 2 & 3 & 4 & 5 & 6 & 7 & 8 & 9 \\
\hline Cx I & 1 & 1 & 1 & 1 & 1 & 1 & 1 & 1 & 1 \\
Cx II & 1 & 1 & 1 & 1 & 1 & 1 & 1 & 1 & 1 \\
Cx III & 1 & 1 & 1 & 1 & 1 & 1 & 1 & 1 & 1 \\
Tr I & 2 & 2 & 2 & 2 & 2 & 2 & 2 & 2 & 2 \\
Tr II & 2 & 2 & 2 & 2 & 2 & 2 & 2 & 2 & 2 \\
Tr III & 2 & 2 & 2 & 2 & 2 & 2 & 2 & 2 & 2 \\
BFe I & 4 & 3 & 5 & 5 & 4 & 5 & 4 & 4 & 4 \\
BFe II & 4 & 4 & 5 & 5 & 4 & 5 & 4 & 4 & 4 \\
BFe III & 4 & 4 & 5 & 5 & 4 & 5 & 4 & 4 & 4 \\
TFe I & 7 & 7 & 8 & 8 & 9 & 8 & 7 & 8 & 5 \\
TFe II & 5 & 5 & 5 & 5 & 9 & 5 & 5 & 6 & 5 \\
TFe III & 5 & 5 & 5 & 5 & 8 & 5 & 5 & 6 & 5 \\
Ge I & 9 & - & 11 & 11 & 13 & 11 & 10 & 11 & 8 \\
Ge II & 9 & 8 & 9 & 9 & 10 & 9 & 9 & 9 & 8 \\
Ge III & 9 & 9 & 9 & 9 & 10 & 9 & 8 & 9 & 8 \\
\hline
\end{tabular}


Table 2 (continued)

\begin{tabular}{|c|c|c|c|c|c|c|c|c|c|}
\hline Character & 1 & 2 & 3 & 4 & 5 & 6 & 7 & 8 & 9 \\
\hline Ti I & 12 & 14 & 14 & 13 & 19 & 13 & 12 & 15 & 10 \\
\hline Ti II & 12 & 15 & 13 & 13 & 17 & 13 & 12 & 13 & 10 \\
\hline Ti III & 12 & 10 & 13 & 11 & 16 & 13 & 12 & 13 & 10 \\
\hline Ta I* & 17 & 26 & 26 & 25 & 26 & 21 & 18 & 23 & 15 \\
\hline Ta II* & 15 & 19 & 22 & 20 & 21 & 22 & 12 & 20 & 13 \\
\hline Ta III* & 18 & 18 & 25 & 22 & 22 & 22 & 14 & 21 & 17 \\
\hline СрфТі I & 0 & 0 & 0 & 0 & 0 & 0 & 0 & 0 & 0 \\
\hline Сp $\zeta$ Ta I & 0 & 0 & 0 & 1 & 1 & 0 & 0 & 0 & 0 \\
\hline Сp $\zeta$ Ta II & 0 & 0 & 0 & 1 & 1 & 0 & 0 & 0 & 0 \\
\hline$\zeta$ Ta I & 2 & - & 2 & 2 & 2 & 2 & 2 & 2 & 2 \\
\hline$\zeta$ Ta II & 1 & - & 2 & 2 & 2 & 2 & 2 & 2 & 1 \\
\hline ЂТa III & 0 & - & 0 & 1 & 1 & 1 & 2 & 1 & 0 \\
\hline$\varepsilon \mathrm{Ta} \mathrm{I}$ & 0 & 1 & 1 & 1 & 1 & 1 & 0 & 1 & 0 \\
\hline$\varepsilon$ Ta II & 0 & 0 & 0 & 1 & 1 & 0 & 0 & 0 & 0 \\
\hline$\kappa \mathrm{Ge} I$ & 1 & - & 1 & 1 & 1 & 1 & 1 & 1 & 0 \\
\hline$\kappa \mathrm{Ge}$ II & 1 & 0 & 1 & 1 & 1 & 1 & 1 & 1 & 0 \\
\hline$\kappa \mathrm{Ti} \mathrm{I}$ & 1 & 1 & 1 & 1 & 1 & 1 & 1 & 1 & 0 \\
\hline$\kappa \mathrm{Ti}$ II & 0 & 1 & 0 & 0 & 0 & 0 & 1 & 0 & 0 \\
\hline$\sigma \mathrm{Ge} I$ & 1 & - & 1 & 1 & 1 & 1 & 1 & 1 & 1 \\
\hline$\sigma \mathrm{Ge} I I$ & 1 & 1 & 1 & 1 & 1 & 1 & 1 & 1 & 0 \\
\hline$\sigma \mathrm{Ge}$ III & 1 & 1 & 1 & 1 & 1 & 0 & 1 & 1 & 0 \\
\hline$\phi T i \mathrm{I}$ & 2 & 1 & 2 & 2 & 2 & 2 & 2 & 2 & 2 \\
\hline$\phi \mathrm{Ti}$ II & 2 & 1 & 2 & 2 & 2 & 2 & 2 & 2 & 2 \\
\hline$\phi \mathrm{Ti}$ III & 1 & 1 & 1 & 1 & 1 & 1 & 1 & 1 & 1 \\
\hline$\omega \mathrm{Ta} \mathrm{I}$ & 1 & 1 & 1 & 1 & 1 & 1 & 1 & 1 & 1 \\
\hline$\omega \mathrm{Ta}$ II & 1 & 1 & 1 & 1 & 1 & 1 & 1 & 1 & 1 \\
\hline$\omega$ Ta III & 0 & 0 & 0 & 0 & 0 & 0 & 0 & 0 & 0 \\
\hline Palpaltibia & 3 & $1 ?$ & 3 & 3 & 3 & 3 & 3 & 3 & 3 \\
\hline Palpalgenu & $2 \mathrm{~N}$ & $2 B$ & $1 \mathrm{~B}, 1 \mathrm{~N}$ & $2 B$ & $2 B$ & $2 B$ & $2 \mathrm{~B}$ & $2 B$ & $2 \mathrm{~N}$ \\
\hline Palpalfemur & $1 \mathrm{~B}, 1 \mathrm{~N}$ & $1 \mathrm{~B}, 1 \mathrm{~N}$ & $1 \mathrm{~B}, 1 \mathrm{~N}$ & $2 \mathrm{~B}$ & $2 \mathrm{~B}$ & $2 \mathrm{~B}$ & $2 \mathrm{~B}$ & $2 \mathrm{~B}$ & $2 \mathrm{~N}$ \\
\hline $\mathrm{fD}(\mathrm{HT})$ & 96 & 40 & 119 & $>140$ & 42 & 120 & - & 60 & 46 \\
\hline
\end{tabular}

* These data have variation usually within the larger series of specimens but there are not any variation in $M$. sanandajensis sp. $n$. because only 5 specimens were collected and studied. 
Table 3. Measurements of nine species of the genus Marantelophus: $1=$ M. alaperti, $2=M$. ostovani, $3=M$. haitlingeri, $4=M$. multisetosa, $5=M$. bella, $6=M$. kamalii, $7=M$. ainae, $8=M$. sanandajensis sp. n., 9. M. emanueli.

\begin{tabular}{|c|c|c|c|c|c|c|c|c|c|}
\hline $\begin{array}{l}\text { Charac- } \\
\text { ter }\end{array}$ & 1 & 2 & 3 & 4 & 5 & 6 & 7 & 8 & 9 \\
\hline AW & 38 & 42 & 70 & $39-48$ & - & $44-47$ & 40 & $35-47$ & $28-32$ \\
\hline PW & 48 & 52 & 82 & $47-57$ & - & 60 & $54-58$ & $53-69$ & $44-52$ \\
\hline AA & - & 14 & 19 & $12-13$ & 13 & 14 & 16 & $10-12$ & 8 \\
\hline SB & - & 16 & 16 & $10-12$ & 36 & $13-14$ & $12-16$ & $12-15$ & 10 \\
\hline $\mathrm{AP}$ & - & 24 & 19 & $12-18$ & 25 & 14-19 & 24 & $17-23$ & $18-22$ \\
\hline $\mathrm{AL}$ & 58 & 56 & 54 & $25-32$ & 28 & 36 & $30-34$ & $23-27$ & 22 \\
\hline PL & 82 & 40 & 67 & $38-43$ & 45 & $44-49$ & $32-36$ & $33-42$ & $28-30$ \\
\hline $\mathrm{AM}$ & - & 36 & 46 & $32-35$ & 52 & $40-41$ & $22-26$ & $17-30$ & $28-30$ \\
\hline$S$ & - & - & 52 & $53-58$ & 43 & 69-77 & $48-54$ & $48-62$ & 32 \\
\hline SD & 70 & 74 & 83 & $49-57$ & 72 & $58-61$ & $70-72$ & $58-69$ & $54-62$ \\
\hline W & - & 90 & 86 & $52-62$ & 77 & 66 & $62-64$ & $62-74$ & $46-58$ \\
\hline ISD & 38 & 56 & 56 & $31-37$ & 50 & 41 & $48-50$ & $44-52$ & $40-44$ \\
\hline GL & 140 & 110 & - & - & - & 124-192 & $106-110$ & 85-109 & $88-94$ \\
\hline $1 a$ & - & 42 & 52 & $24-34$ & 38 & $22-25$ & $26-30$ & $27-40$ & 20 \\
\hline $1 b$ & - & 44 & 60 & $25-43$ & 49 & $41-46$ & $42-44$ & $38-53$ & $30-36$ \\
\hline $2 b$ & - & 34 & 40 & $20-36$ & 38 & $33-36$ & $28-34$ & $21-38$ & 18 \\
\hline $3 b$ & - & 38 & 32 & $19-33$ & 28 & 22 & $26-28$ & $20-25$ & $18-20$ \\
\hline PaScFed & - & 36 & - & - & - & 38 & $38-44$ & $33-47$ & 34 \\
\hline $\mathrm{PaScFev}$ & - & 30 & - & - & - & $44-49$ & & $40-54$ & - \\
\hline PaScGed & - & 26 & - & - & - & $22-33$ & $26-28$ & $20-29$ & - \\
\hline PaScGev & - & 20 & - & - & - & 33 & - & $25-28$ & - \\
\hline Ta I (L) & 80 & 62 & 100 & $65-72$ & 71 & $74-77$ & $50-54$ & $52-62$ & 48 \\
\hline Ta I (H) & - & - & - & - & - & $21-24$ & $28-30$ & $25-27$ & - \\
\hline $\mathrm{Ti} \mathrm{I}$ & - & 82 & 106 & $56-70$ & 89 & $69-74$ & $68-74$ & $58-67$ & $54-58$ \\
\hline Ge I & - & - & 90 & $57-73$ & 73 & $74-77$ & $70-72$ & $55-67$ & $50-54$ \\
\hline TFe I & - & 44 & 50 & $36-43$ & 45 & $36-38$ & $34-36$ & $28-37$ & 30 \\
\hline BFe I & - & 42 & 60 & $45-51$ & 52 & $44-47$ & $42-48$ & $30-40$ & $30-38$ \\
\hline $\operatorname{Tr} I$ & - & 40 & 50 & $38-45$ & 41 & 42 & $32-34$ & $30-37$ & $30-32$ \\
\hline Cx I & - & 52 & 70 & - & - & $50-52$ & $54-60$ & $43-50$ & $46-50$ \\
\hline Leg I & - & - & 526 & 356-398 & 433 & - & $360-368$ & $305-348$ & $292-306$ \\
\hline Ta II (L) & - & 60 & 84 & $63-65$ & 66 & $66-74$ & 48 & $45-52$ & 44 \\
\hline
\end{tabular}


Table 3 (continued)

\begin{tabular}{lccccccccc}
\hline $\begin{array}{l}\text { Charac- } \\
\text { ter }\end{array}$ & 1 & 2 & 3 & 4 & 5 & 6 & 7 & 8 & 9 \\
\hline Ta II (H) & - & 28 & - & - & - & $18-24$ & 24 & $22-23$ & - \\
Ti II & - & 84 & 100 & $58-68$ & 79 & $63-74$ & $60-62$ & $55-59$ & $48-52$ \\
Ge II & - & 70 & 78 & $58-65$ & 60 & $66-69$ & $56-60$ & $50-62$ & $44-50$ \\
TFe II & - & 40 & 40 & $32-35$ & 39 & $30-33$ & 32 & $25-35$ & $24-26$ \\
BFe II & - & 44 & 50 & $42-48$ & 43 & $36-41$ & $34-38$ & $30-38$ & $22-32$ \\
Tr II & - & 42 & 57 & $41-47$ & 39 & $36-41$ & 40 & $32-40$ & $30-34$ \\
Cx II & - & 64 & 88 & - & - & 55 & $66-72$ & $50-62$ & 42 \\
Leg II & - & - & 497 & $321-377$ & 386 & - & $338-350$ & $293-345$ & 276 \\
Ta III (L) & 84 & 62 & & $66-72$ & 73 & $74-77$ & $48-52$ & $45-54$ & \\
Ta III (H) & - & 22 & 90 & - & - & $19-22$ & 22 & $17-23$ & - \\
Ti III & 112 & 102 & - & $76-106$ & 112 & $96-102$ & $82-84$ & $70-77$ & 72 \\
Ge III & - & 90 & 140 & $69-79$ & 78 & $77-79$ & 72 & $58-71$ & \\
TFe III & - & 42 & 96 & $38-49$ & 52 & $41-44$ & $44-46$ & $30-42$ & \\
BFe III & - & 50 & 54 & $47-64$ & 56 & 47 & $44-46$ & $37-42$ & \\
Tr III & - & 46 & 60 & $41-50$ & 42 & $41-42$ & $42-46$ & $37-47$ & 32 \\
Cx III & - & 70 & 60 & - & - & 58 & $64-67$ & $47-62$ & \\
Leg III & & & 80 & & & & - & $332-343$ & \\
IP & & & & & & & - & $930-1086$ & \\
\hline & & & & & & & & &
\end{tabular}

Table 4. Host of 9 species of the genus Marantelophus.

\begin{tabular}{ll}
\hline Species & Host \\
\hline M. alaperti & off host \\
M. ostovani & undetermined Aphididae \\
M. haitlingeri & undetermined Heteroptera \\
M. multisetosa & Thrips tabaci \\
M. bella & Sericothrips sp. \\
M. kamalii & off host \\
M. ainae & off host \\
M. sanandajensis sp. n. & unidentified Thysanoptera and Aphididae \\
M. emanueli & off host \\
\hline
\end{tabular}


Acknowledgements - This research was supported by a grant from University of Kurdistan and "Center of Excellence of Biological Control of Pests" Department of Plant Protection, Faculty of Agriculture, University of Tehran, Karaj, Iran, which is greatly appreciated. The authors also thanks to F. Afrasiabi for helping on sampling.

\section{REFERENCES}

Goldarazena, A. \& Zhang, Z. Q. (1997) First record of larval Grandjeanella (Acari: Erythraeidae) from Heteroptera and description of a new species from Spain. Systematic $\mathcal{E}$ Applied Acarology 2: 231-236.

Haitlinger, R. (2002) Erythraeidae and Trombidiidae (Allothrombiinae) (Acari: Prostigmata from Mallorca (Balearic Islands), with description of two new species. Bolletin de la Sociedad de Historia Natural de Baleares 45: 191-197.

Haitlinger, R. (2010) New records of mites (Acari: Prostigmata: Erythraeidae, Trombidiidae) from Turkey, with descriptions of four new species. Zeszyty Naukowe Uniwersytetu Przyrodniczego we Wrocławiu, Biologia i Hodowla Zwierzat 60(577): 49-61.

Haitlinger, R. (2011) A new genus and four new species of erythraeid mites from Indonesia, with new records of the family (Acari: Prostigmata: Erythraeidae). Revista Ibérica de Aracnología 19: 47-54.

Haitlinger, R. \& Saboori, A. (1996) Seven new larval mites (Acari, Prostigmata, Erythraeidae) from Iran. Miscellània Zoològica 19: 117-131.

Mąkol, J. \& Wohltmann, A. (2012) An annotated checklist of terrestrial Parasitengona (Actinotrichida: Prostigmata) of the world, excluding Trombiculidae and Walchiidae. Annales Zoologici 62(3): 359-562. doi: 10.3161/000345412X656671

Saboori, A. \& Atamehr, A. (2000) Grandjeanella kamalii sp. n. (Acari : Erythraeidae) from Iran with a key to world species. Systematic \& Applied Acarology Special Publications 5: 1-8. doi: 10.11158/saasp.5.1.1

Saboori, A., Khaustov, A., Hakimitabar, M. \& Hajiqanbar, H. (2009) A new genus and species of larval Erythraeinae (Acari: Prostigmata: Erythraeidae) from Ukraine and the taxonomic state of Zhangiella. Zootaxa 2203: 22-30.

Walter, D. E. \& Krantz, G. W. (2009) Collecting, rearing, and preparing specimens. Pp. 83-96. In: Krantz, G. W. \& Walter, D. E. (eds): A manual of Acarology, 3rd ed., Texas Tech University Press, Texas.

Wohltmann, A. (2000) The evolution of life histories in Parasitengona (Acari: Prostigmata). Acarologia 41 (1-2): 145-204.

Wohltmann, A. (2001) Closely related species of Parasitengonae (Acari: Prostigmata) inhabiting the same area: Patterns facilitating coexistence. Pp. 121-135. In: Halliday, R. B., Walter, D. E., Proctor, H. C., Norton, R. A. \& Colloff, M. J. (eds): Acarology: Proceedings of the 10th International Congress of Acarology. CSIRO Publishing, Melbourne.

Zhang, Z.-Q. \& Goldarazena, A. (1996) Abrolophus and Grandjeanella larvae (Acari: Erythraeidae) ectoparasitic on thrips (Thysanoptera: Thripidae). Systematic \& Applied Acarology 1: 127-144.

Revised version received January 21, 2015, accepted May 17, 2015, published August 14, 2015 